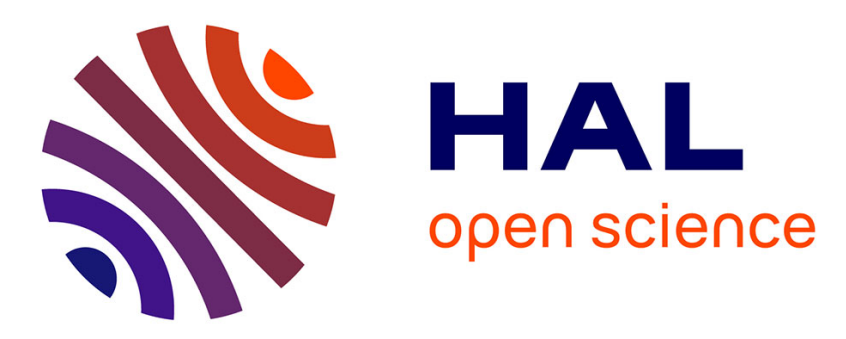

\title{
3D surface measurements with isogeometric stereocorrelation: Application to complex shapes
}

John-Eric Dufour, Sylvain Leclercq, Julien Schneider, Stéphane Roux, François Hild

\section{- To cite this version:}

John-Eric Dufour, Sylvain Leclercq, Julien Schneider, Stéphane Roux, François Hild. 3D surface measurements with isogeometric stereocorrelation: Application to complex shapes. Optics and Lasers in Engineering, 2016, 87, pp.146 - 155. 10.1016/j.optlaseng.2016.02.018 . hal-01383958

\section{HAL Id: hal-01383958 \\ https://hal.science/hal-01383958}

Submitted on 19 Oct 2016

HAL is a multi-disciplinary open access archive for the deposit and dissemination of scientific research documents, whether they are published or not. The documents may come from teaching and research institutions in France or abroad, or from public or private research centers.
L'archive ouverte pluridisciplinaire HAL, est destinée au dépôt et à la diffusion de documents scientifiques de niveau recherche, publiés ou non, émanant des établissements d'enseignement et de recherche français ou étrangers, des laboratoires publics ou privés. 


\title{
3D surface measurements with isogeometric stereocorrelation - Application to complex shapes
}

\author{
John-Eric Dufour ${ }^{\mathrm{a}, \mathrm{b}}$, Sylvain Leclercq ${ }^{\mathrm{c}}$, Julien Schneider $^{\mathrm{b}}$, Stéphane Roux ${ }^{\mathrm{a}}$, \\ François Hild ${ }^{\mathrm{a}, *}$ \\ ${ }^{a}$ LMT-Cachan, ENS Cachan / CNRS / Université Paris-Saclay, 61 Avenue du Président Wilson \\ 94235 Cachan Cedex, France \\ ${ }^{b}$ SAFRAN Group, Snecma site Villaroche, YQMC, Rond point René RAVAUD, 77550 \\ Moissy-Cramayel, France \\ ${ }^{c}$ SAFRAN Group, Messier-Bugatti-Dowty, Inovel Parc Sud, 78140 Vélizy Villacoublay, France
}

\begin{abstract}
The aim of the present study is to measure complex shapes of tested objects by using a priori information given by their CAD model via stereocorrelation. To follow a 3D object during its deformation and to determine 3D surface displacement fields, a first measurement of the object shape is necessary. It is achieved by updating the $\mathrm{CAD}$ reference via a global approach to stereocorrelation. Once the 3D shape has been determined, the next step is to measure 3D displacement fields during loading. The kinematics of the deformed shape is assumed to be written within the same isogeometric framework. Isogeometric stereocorrelation is applied to analyze a compression test on a ribbed cylinder in two different configurations of the stereo rig.
\end{abstract}

Keywords: Stereocorrelation, freeform surfaces, displacement

\footnotetext{
${ }^{*}$ Corresponding Author

Email address: hild@lmt.ens-cachan.fr (François Hild)
} 


\section{Introduction}

Many techniques are presently used to measure the 3D external shape of an object. 3D scanners either probe the surface of interest by physical contact (e.g., coordinate measuring machine), emit radiations and detect their reflection on the surface (e.g., time-of-flight 3D laser scanners, triangulation-based 3D laser scanners), or project structured light and analyze the deformation of the fringes [1]. Last, non-contact passive scanners detect reflected ambient radiations (e.g., stereoscopic systems [2, 3, 4, 5]). All these 3D scanners evaluate the position of some points on the surface and this cloud is further interpolated to obtain a continuous representation. These techniques involve multitudinous degrees of freedom during the point cloud evaluation, which is subsequently reduced by interpolation without a posteriori control of the quality. The present approach consists of adding as much a priori information as possible to measure the 3D shape of the surface and assessing the quality of the reconstruction. In the case of industrial parts, the CAD model of the nominal shape is generally available and can be used as a first estimate. By resorting to global stereocorrelation, this surface can be deformed to fit as well as possible (i.e., by minimizing the correlation residuals) the real shape [6].

Shape reconstruction via stereocorrelation consists of finding the 3D coordinates of a point using its $2 \mathrm{D}$ coordinates in 2 (or more) pictures shot by cameras. This reconstruction assumes that the calibration of the stereovision system has already been performed [7, 8, 9]. Classical (i.e., local) approaches perform this operation by resorting to spatial registrations to extract a cloud of $3 \mathrm{D}$ points from $2 \mathrm{D}$ pictures. 
The method developed herein does not reconstruct point to point surfaces but uses Non-Uniform Rational Basis Splines (or NURBS [10]) to measure the 3D shape as a freeform representation without any need for subsequent interpolation.

Once the initial 3D shape has been determined, the next step is to follow its deformation during, say, a mechanical test. Classical stereocorrelation systems provide the user with point clouds from which the 3D displacements are obtained by resorting to temporal registrations [9]. An alternative to the former approach, which will be followed herein, is to describe the surface deformation using a specific parameterization and to determine those parameters in time. Among the many possible representations of surface deformation, a natural choice is the NURBS framework itself, namely, the motion of control points defining the initial 3D shape [11]. Let us note that this choice is not restrictive as it may appear as one can refine the shape description with the introduction of additional control points. In the opposite limit, too many degrees of freedom may lead to ill-conditioning and high noise sensitivity. This issue will be illustrated in the present study. Last, the observed 3D shape may have complex features such as very sharp angles (e.g., see [12]) or areas with small radii of curvatures (e.g., ribs). In these difficult cases, standard reconstruction methods may fail [12]. This particular point will also be addressed herein to analyze a compression test on the upper diaphragm tube of a landing gear.

The outline of the paper is as follows. First, the isogeometric framework is introduced when surfaces are described by NURBS. The main equations of the global stereocorrelation procedure are detailed to reconstruct the initial 3D shape of the surface of interest. This procedure is first applied to study a complex shape con- 
taining small scale features with sharp edges and for which not all information is available on both cameras. The stereocorrelation technique is driven through a novel regularization scheme, which limits the effect of the poor conditioning induced by hidden parts, and allows the code to converge toward an acceptable solution. To speed up the computations, a new multiscale algorithm is implemented. Last, 3D displacement fields in a compressed ribbed cylinder are measured when the stereo rig has been moved to a more natural position.

\section{Surface representation with NURBS}

In this study, the starting point of the analyzed surface is its nominal CAD model. Its geometry is a freeform surface made of NURBS patches [10]. Nowadays most parts have a CAD representation based on this type of model [13]. The latter provides a generic and standard representation of complex shapes with fewer degrees of freedom than standard meshes.

A NURBS patch is defined by i) its order, ii) a network of control points with associated weights, and iii) its knot vector (Figure 5(b)). The surface $\mathbf{X}(u, v)=$ $(X, Y, Z)$ is expressed in the parametric space $(u, v)$ as

$$
\mathbf{X}(u, v)=\frac{\sum_{i=0}^{m} \sum_{j=0}^{n} N_{i, p}(u) N_{j, q}(v) \omega_{i j} \mathbf{P}_{\mathbf{i j}}}{\sum_{i=0}^{m} \sum_{j=0}^{n} N_{i, p}(u) N_{j, q}(v) \omega_{i j}}
$$

with

$$
\forall u \in[0,1], N_{i, 0}(u)=\left\{\begin{array}{l}
1 \text { when } u_{i} \leq u \leq u_{i+1} \\
0 \text { otherwise }
\end{array}\right.
$$

and

$$
N_{i, p}(u)=\frac{u-u_{i}}{u_{i+p}-u_{i}} N_{i, p-1}(u)+\frac{u_{i+p+1}-u}{u_{i+p+1}-u_{i+1}} N_{i+1, p-1}(u)
$$


where $N_{i, p}$ are mixing functions, $\mathbf{P}_{\mathbf{i j}}$ the coordinates of control points of the surface, $\omega_{i j}$ the corresponding weights, $(m+1) \times(n+1)$ the number of control points and $(p, q)$ the degrees of the surface.

Figure 1(a) shows the CAD model of a landing gear. The red arrow points to the location of the studied part once mounted. A picture of the upper diaphragm that was prepared for correlation purposes (i.e., a random pattern was created by spraying $\mathrm{B} / \mathrm{W}$ paint) is shown in Figure 1(b). In the present case it is made of injected PEEK reinforced by short carbon fibers. This type of structure is found in landing gears. It is worth noting that 8 ribs are equally spaced along the circumferential direction. The measurement of the 3D shape becomes very challenging because of the presence of these ribs. The CAD model of this shape is composed of $18 \times 4$ control points with

fourth order shape functions (see Figure 5(b)). In the present case all the weights of the control points are chosen to be 1 . It is worth noting that there is a large number of points on the rib to be able to describe its sharpness faithfully.

\section{3D shape measurements via isogeometric stereocorrelation}

In the following, the calibration and 3D reconstruction of the reference configuration of the studied shape is performed by resorting to a self-calibration procedure [7, 14]. This is of particular interest for structures for which the calibration via targets may become cumbersome if not impossible. The first step is to calibrate the stereoscopic setup by determining the projection matrices. Once the latter ones are known, the freeform definition of the surface of interest is matched as well as possible to the actual 3D shape. These two steps are controlled by analyzing the correlation 


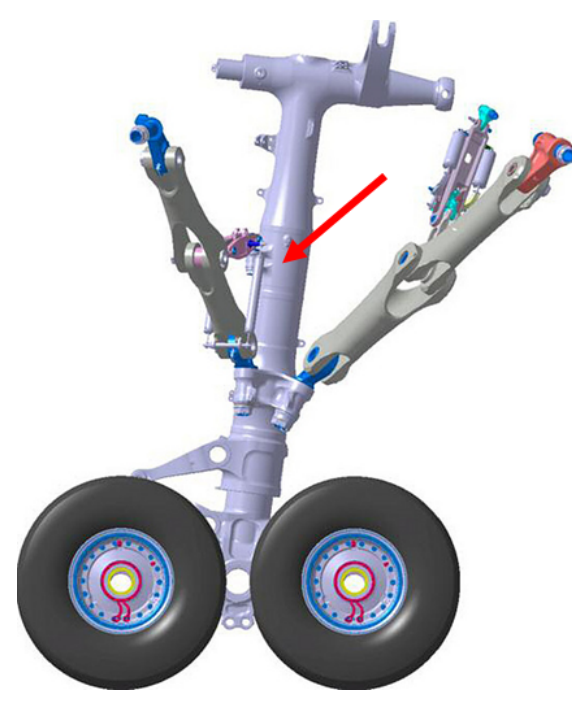

(a)

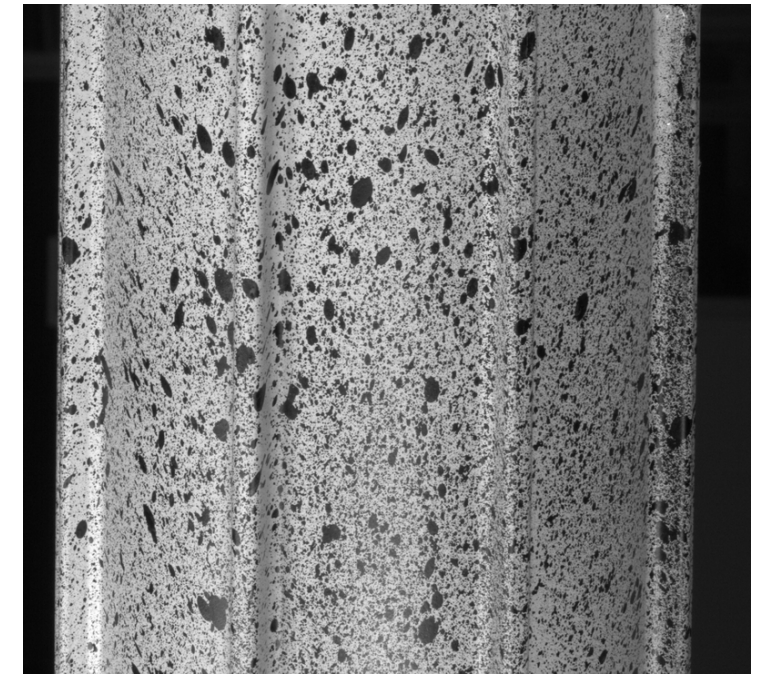

(b)

Figure 1: (a) Landing gear. The red arrow points to the location of the upper diaphragm, which is an internal part. (b) Picture of the observed surface for which 4 ribs are visible

residuals.

\subsection{Calibration of the stereoscopic system}

The calibration of the stereoscopic system is achieved by using a global approach to stereocorrelation [6]. In the present case, the object of interest will serve as calibration target since its nominal shape is known. First the projection matrices [15, 9 for the left (i.e., $\left[\mathbf{M}^{l}\right]$, which is a $3 \times 4$ matrix) and right (i.e., $\left[\mathbf{M}^{r}\right]$, a second $3 \times 4$ matrix) cameras are calibrated by resorting to integrated DIC [16], which consists of minimizing the sum of squared differences (Figure 2)

$$
\eta^{2}\left(\left[\mathbf{M}^{l, r}\right]\right)=\sum_{\mathrm{ROI}}\left(f^{l}\left(\mathbf{x}^{l}\left(u, v,\left[\mathbf{M}^{l}\right]\right)\right)-f^{r}\left(\mathbf{x}^{r}\left(u, v,\left[\mathbf{M}^{r}\right]\right)\right)\right)^{2}
$$


with respect to each unknown matrix component $M_{i j}^{r, l}$, where the homogeneous coordinates of any $3 \mathrm{D}$ point $\{\overline{\mathbf{X}}\}=(X, Y, Z, 1)^{\dagger}$ are related to the corresponding left and right homogeneous coordinates $\left(\left\{\overline{\mathbf{x}^{l}}\right\}=\left(s^{l} x^{l}, s^{l} y^{l}, s^{l}\right)^{\dagger}\right.$ and $\left\{\overline{\mathbf{x}^{r}}\right\}=\left(s^{r} x^{r}, s^{r} y^{r}, s^{r}\right)^{\dagger}$, by the two projection matrices [17]

$$
\left\{\overline{\mathbf{x}^{l}}\right\}=\left[\mathbf{M}^{l}\right]\{\overline{\mathbf{X}}\} \quad \text { and } \quad\left\{\overline{\mathbf{x}^{r}}\right\}=\left[\mathbf{M}^{r}\right]\{\overline{\mathbf{X}}\}
$$

where $s^{l}$ and $s^{r}$ are scale factors, ${ }^{\dagger}$ the transposition operator, and $f^{l, r}$ the left and right pictures in the reference configuration. In the present setting, the sensitivity fields, $\partial \mathbf{x}^{i} / \partial\left[\mathbf{M}^{i}\right]$ (with $i=l$ or $r$ ), with respect to each unknown component are used to compute the new coordinates induced by small increments $\delta\left[\mathbf{M}^{l}\right]$ and $\delta\left[\mathbf{M}^{r}\right]$

$$
\begin{aligned}
\mathbf{x}^{l}\left(\left[\mathbf{M}^{l}\right]+\left[\delta \mathbf{M}^{l}\right]\right) & =\mathbf{x}^{l}\left(\left[\mathbf{M}^{l}\right]\right)+\frac{\partial \mathbf{x}^{l}}{\partial\left[\mathbf{M}^{l}\right]}\left(\left[\mathbf{M}^{l}\right]\right) \delta\left[\mathbf{M}^{l}\right] \\
\mathbf{x}^{r}\left(\left[\mathbf{M}^{r}\right]+\left[\delta \mathbf{M}^{r}\right]\right) & =\mathbf{x}^{r}\left(\left[\mathbf{M}^{r}\right]\right)+\frac{\partial \mathbf{x}^{r}}{\partial\left[\mathbf{M}^{r}\right]}\left(\left[\mathbf{M}^{r}\right]\right) \delta\left[\mathbf{M}^{r}\right]
\end{aligned}
$$

where the dependence on the parametric coordinates $(u, v)$ has been omitted for the sake of conciseness. Consequently, the vectors $\left\{\delta \mathbf{x}^{l, r}\right\}$ gathering all the variations $\delta \mathbf{x}^{l, r}$ when $(u, v)$ span over the parametric space read

$$
\left\{\delta \mathbf{x}^{l, r}\right\}=\left[\mathbf{S}_{\mathbf{M}}\right]\{\delta \mathbf{m}\}
$$

where $\{\delta \mathbf{m}\}$ gathers 22 out of 24 unknown components of the projection matrices $M_{i j}^{r, l}$, and $\left[\mathbf{S}_{\mathbf{M}}\right]$ is the sensitivity matrix whose lines span over all the considered values of the parametric space (i.e., $n_{u} \times n_{v}$ ) and the number of rows is equal to 22. The two remaining unknowns have to be determined from the knowledge of an absolute dimension in the observed structure [6]. 


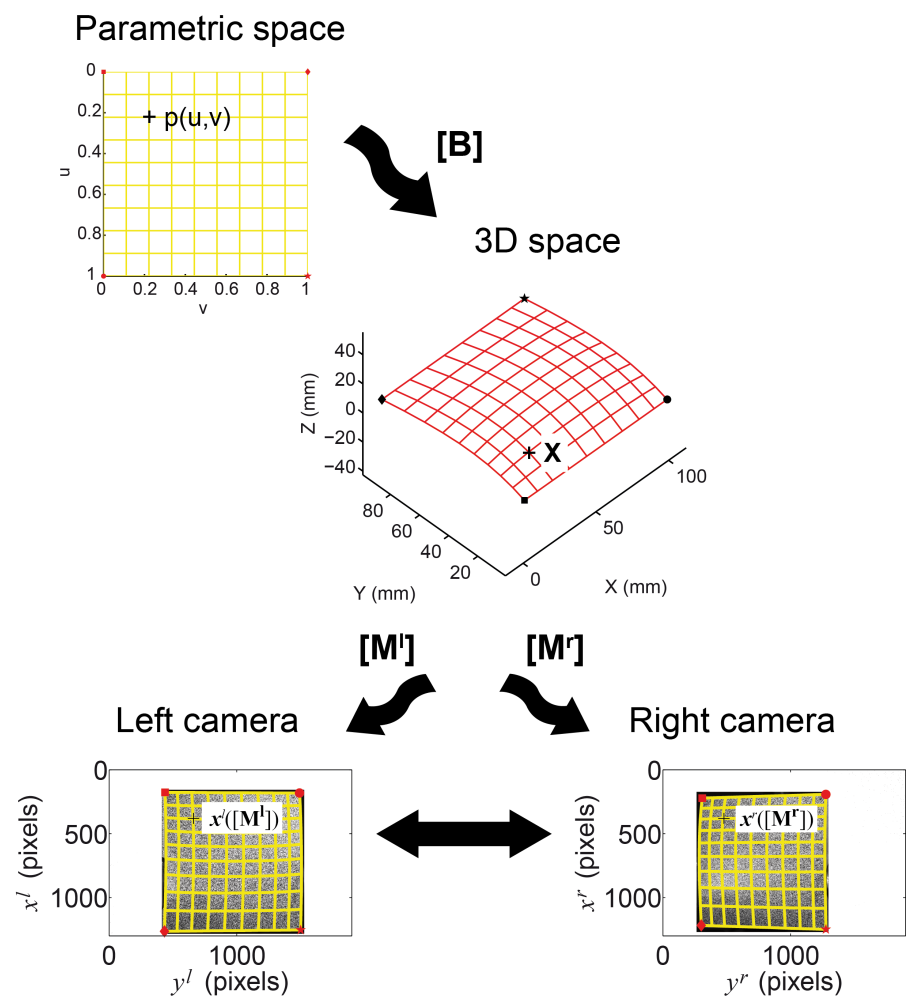

Figure 2: Determination of the projection matrices via isogeometric stereocorrelation

The previous minimization is achieved by resorting to Newton-Raphson scheme in which linearizations and corrections are performed [18]. The following linear system is solved to determine the corrections $\{\delta \mathbf{m}\}$

$$
\left[\mathbf{C}_{\mathbf{M}}\right]\{\delta \mathbf{m}\}=\left\{\mathbf{b}_{\mathbf{M}}\right\}
$$

where $\left[\mathbf{C}_{\mathbf{M}}\right]$ denotes the $(22 \times 22)$ stereocorrelation matrix

$$
\left[\mathbf{C}_{\mathbf{M}}\right]=\left[\mathbf{G}_{\mathbf{M}}\right]^{\dagger}\left[\mathbf{G}_{\mathbf{M}}\right]
$$

$\left\{\mathbf{b}_{\mathbf{M}}\right\}$ the stereocorrelation vector

$$
\left\{\mathbf{b}_{\mathbf{M}}\right\}=\left[\mathbf{G}_{\mathbf{M}}\right]^{\dagger}\{\mathbf{r}\}
$$


$\{\mathbf{r}\}$ the vector gathering all correlation residuals for the considered values $(u, v)$ in the parametric space

$$
r(u, v)=f^{l}\left(\mathbf{x}^{l}\left(u, v,\left[\mathbf{M}^{l}\right]\right)\right)-f^{r}\left(\mathbf{x}^{r}\left(u, v,\left[\mathbf{M}^{r}\right]\right)\right)
$$

and $\left[\mathbf{G}_{\mathbf{M}}\right]$ the $\left(n_{u} \times n_{v}\right) \times 22$ matrix collecting all values of the scalar product of the picture gradients $\nabla f^{l, r}(u, v)$ by the sensitivity fields $\partial \mathbf{x}^{l, r} / \partial m_{i}\left(u, v,\left[\mathbf{M}^{l}\right]\right)$.

\subsection{Self-Calibration}

The first guess of the 3D shape (i.e., its nominal freeform) is projected onto the $2 \mathrm{D}$ space using the previously measured projection matrices $\left[\mathbf{M}^{l, r}\right]$ generating two grids associated with positions in the parametric space. Right and left pictures are interpolated through these grids to create two sub-pictures in the parametric space. A global approach to stereocorrelation [6] is performed between these two sub-figures by moving the control points $\mathbf{P}_{\mathbf{i j}}$ of the NURBS patches. These motions induce pseudo displacements in the left and right pictures

$$
\mathbf{x}^{l}=\mathbf{x}_{0}^{l}+\frac{\partial \mathbf{x}^{l}}{\partial \mathbf{X}} \frac{\partial \mathbf{X}}{\partial \mathbf{P}_{\mathbf{i j}}} \delta \mathbf{P}_{\mathbf{i j}} \quad \text { and } \quad \mathbf{x}^{r}=\mathbf{x}_{0}^{r}+\frac{\partial \mathbf{x}^{r}}{\partial \mathbf{X}} \frac{\partial \mathbf{X}}{\partial \mathbf{P}_{\mathbf{i j}}} \delta \mathbf{P}_{\mathbf{i j}}
$$

which induce new sensitivity fields in the same spirit as those introduced above, where $\mathbf{x}_{0}^{l, r}$ denote the initial positions, and $\mathbf{x}^{l, r}$ the corrected ones. This leads to an estimate of the increment of control point positions $\delta \mathbf{P}_{\mathbf{i j}}$ that is to be added to the previous coordinates (Figure 3).

The global stereocorrelation procedure consists of minimizing the sum of squared differences

$$
\eta^{2}\left(\mathbf{P}_{\mathbf{i j}}\right)=\sum_{\mathrm{ROI}}\left(f^{l}\left(\mathbf{x}^{l}\left(u, v, \mathbf{P}_{\mathbf{i j}}\right)\right)-f^{r}\left(\mathbf{x}^{r}\left(u, v, \mathbf{P}_{\mathbf{i j}}\right)\right)\right)^{2}
$$




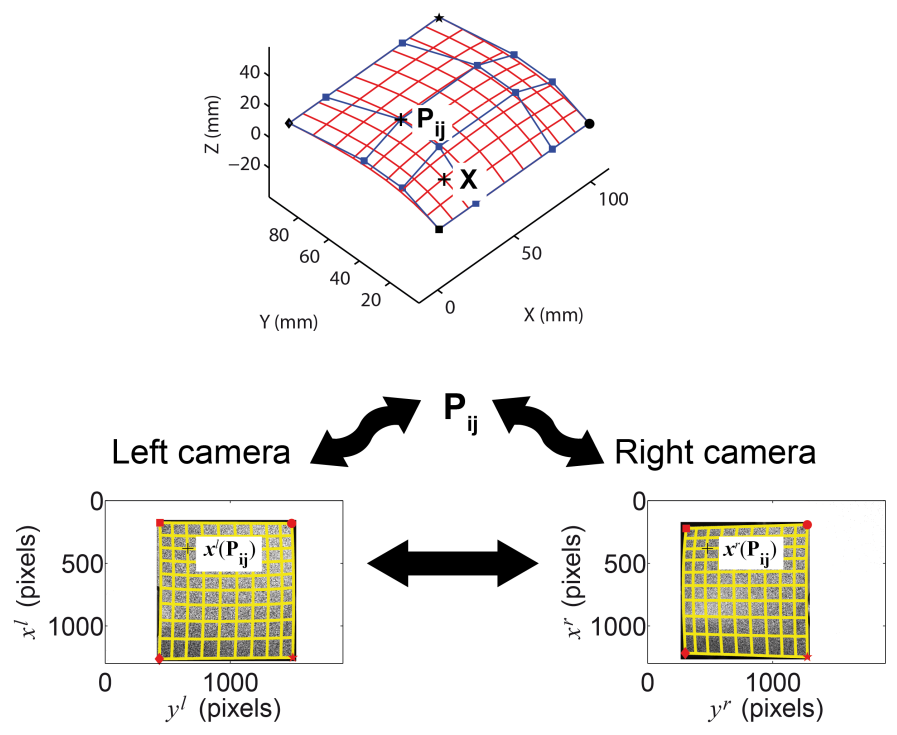

Figure 3: Measurement of the initial 3D shape via isogeometric stereocorrelation

with respect to each unknown position of the control points. Let $\{\delta \mathbf{p}\}$ gather all the control point motions when $(u, v)$ span over the parametric space. The minimization is again achieved by resorting to a Newton-Raphson scheme. The following linear system is solved to determine the corrections $\{\delta \mathbf{p}\}$

$$
\left[\mathbf{C}_{\mathbf{P}}\right]\{\delta \mathbf{p}\}=\left\{\mathbf{b}_{\mathbf{P}}\right\}
$$

where $\left[\mathbf{C}_{\mathbf{P}}\right]$ denotes the $\left(n_{p} \times n_{p}\right)$ stereocorrelation matrix

$$
\left[\mathbf{C}_{\mathbf{P}}\right]=\left[\mathbf{G}_{\mathbf{P}}\right]^{\dagger}\left[\mathbf{G}_{\mathbf{P}}\right]
$$

with $n_{p}$ equal to three times the total number of control points, $\left\{\mathbf{b}_{\mathbf{M}}\right\}$ the stereocorrelation vector

$$
\left\{\mathbf{b}_{\mathbf{P}}\right\}=\left[\mathbf{G}_{\mathbf{P}}\right]^{\dagger}\{\mathbf{r}\}
$$


$\{\mathbf{r}\}$ the vector gathering all correlation residuals

$$
r(u, v)=f^{l}\left(\mathbf{x}^{l}\left(u, v, \mathbf{P}_{\mathbf{i j}}\right)\right)-f^{r}\left(\mathbf{x}^{r}\left(u, v, \mathbf{P}_{\mathbf{i} \mathbf{j}}\right)\right)
$$

and $\left[\mathbf{G}_{\mathbf{P}}\right]$ the $\left(n_{u} \times n_{v}\right) \times n_{p}$ matrix collecting all values of the scalar product of picture gradients $\boldsymbol{\nabla} f^{l, r}(u, v)$ by the sensitivity fields $\partial \mathbf{x}^{l, r} / \partial p_{i}(u, v)$.

It is worth noting that these two steps (i.e., determination of the projection matrices, 3D shape corrections) are to be repeated until final converge is observed. In the present cases, only two iterations were needed.

At convergence, the surface black and white paint speckle pattern that is observed on both views can be transferred to the parametric space. This endows the shape information contained in the CAD model with a texture, which will further allow for the measurement of the surface displacement field not only along its normal but also in its tangent components. This will be the topic of Section 5 .

\section{First application}

The previous method is tested on an industrial part with a complex shape (i.e., upper diaphragm of a landing gear). It consists of a cylinder with 8 vertical ribs (Figure 1(b)). Two digital single-lens reflex cameras (DSLRs) are used (definition: $2601 \times 1733$ pixels, digitization: 8 bits) with 50-mm lenses. Lighting is provided by two LED panels. A third DSLR camera can be seen in Figure 4(a). It has been used to monitor a smaller part outside the region of interest (Figure 4(b)) and will not be discussed herein. In this first application, the stereoscopic setup is oriented, on purpose, in such a way that the left part of the observed rib is not visible by both 
cameras (Figure $4(\mathrm{~b}-\mathrm{c})$ ). It will be shown that the NURBS framework offers a very convenient framework for dealing with such problems through regularization.

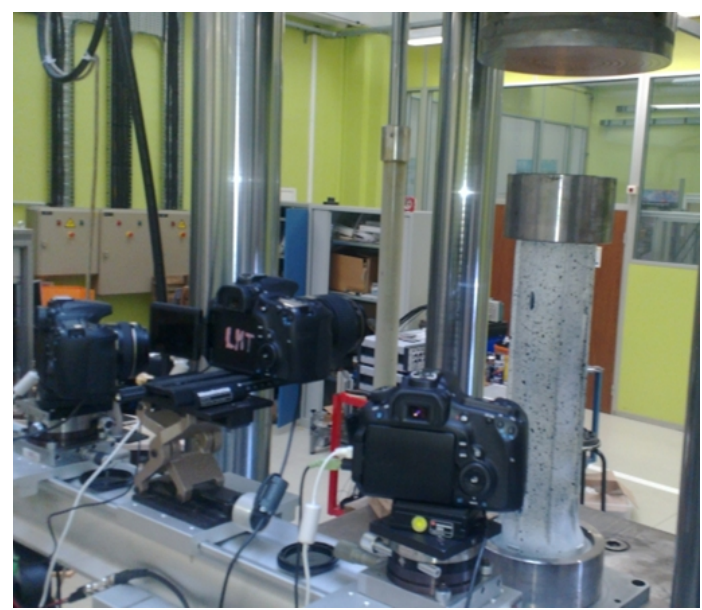

(a)

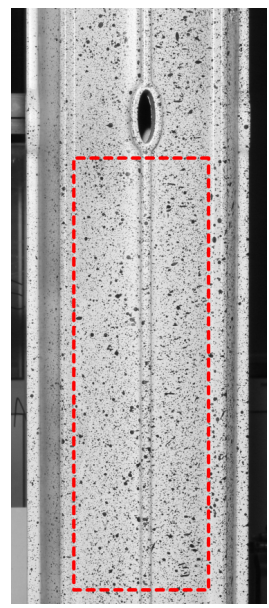

(b)

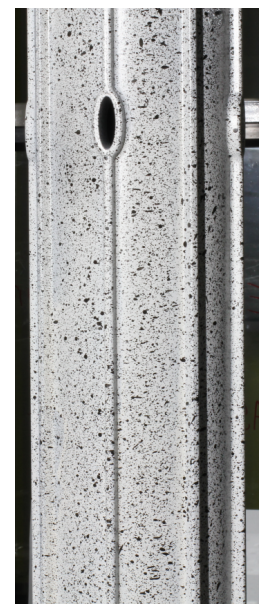

(c)

Figure 4: (a) Stereoscopic setup to monitor the tested upper diaphragm. Corresponding left (b) and right (c) pictures. The reconstructed zone is shown in the red box

\subsection{Direct analysis}

The theoretical shape used in this case is, on purpose, quite distant from the real one and the rib is very smooth compared to the observed one (Figure 5(a)). To perform an accurate reconstruction of the rib, the surface contains more control points on that specific area than in the cylindrical part. Furthermore, only few points are used in the longitudinal direction (i.e., it is assumed that the 3D shape is close to a cylinder whose nominal directrix is shown in Figure 5(b)).

In the present case, different descriptions of the observed surface could have been used. Instead of adding control points (Figure 5(b)), the multiplicity of some of 


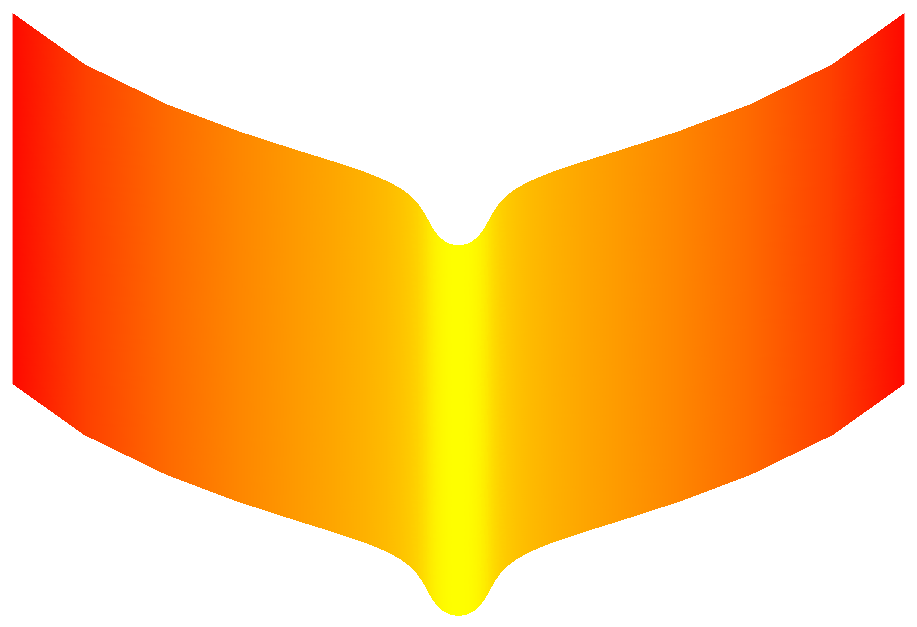

(a)

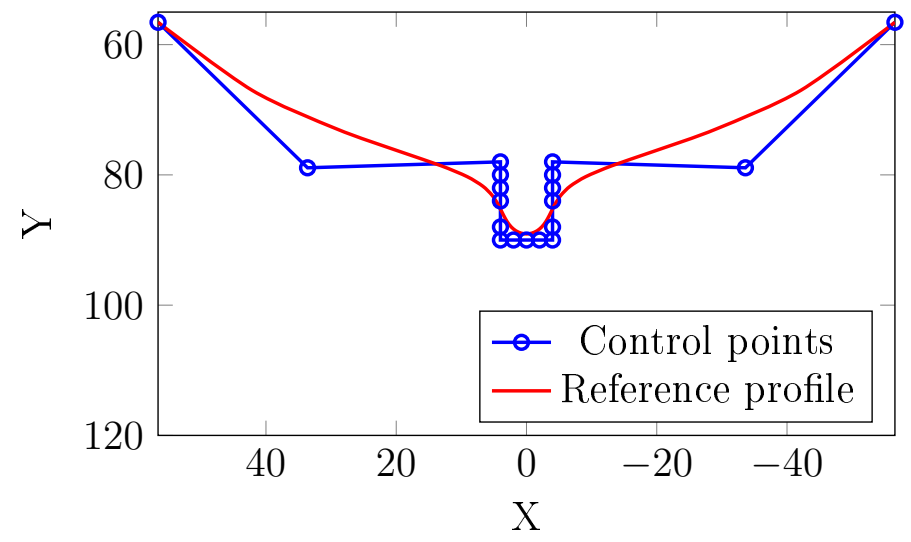

(b)

Figure 5: (a) Initial model of the analyzed area (Figure 4). The color encoding indicates the height with respect to the underlying cylinder. (b) Corresponding directrix of the studied cylinder. The blue circles are control points

them may have been increased in order to tackle the discontinuity of slopes or high curvatures of the ribs. Although an enhanced fidelity to the actual shape would 
definitely promote a better solution, the choice is made herein to deal with an approximate description. As will be seen in the sequel, the poor conditioning induced by the presence of small scale features and hidden parts leads to difficulties. This calls for a regularization strategy whose benefits and limitations will be shown. If a more faithful description had been chosen, the regularization by itself would have been able to achieve an excellent registration, and thus would not be a convincing demonstration of the respective role of stereocorrelation and regularization.

As a side remark, let us also note that other specific mathematical descriptions (i.e., not using NURBS) may have been considered to model the rib (e.g., continuous facets). This may be a very wise choice in practice. However, the present study focuses on a unified isogeometric (i.e., NURBS-based) description of both shapes and displacements, and thus such variants in the description will not be investigated hereafter.

The calibration process of the stereo rig is performed using the self-calibration method described above. It is worth noting that in the present case, two physical dimensions need to be specified because of the particular shape of the object (i.e., invariant along the longitudinal direction). For confidentiality reasons, these absolute dimensions are not given and all the results will be expressed in arbitrary units. Figure 6 shows the theoretical shape used as a first guess during the calibration step. The colors correspond to the residual map prior to (Figure 6(a)) and after (Figure 6(b)) the calibration of the projection matrices. In these maps the gray levels of the registered pictures have been logarithmically re-encoded. As expected, the rib is very distant from the real one so that very high residuals are observed 
after this first calibration step. The RMS value of $r$ is equal to $7.4 \%$ of the dynamic range of the reference pictures after the first step. It is worth noting that even though the initial shape is significantly smoother than the actual one, the calibration of the stereoscopic system is deemed acceptable. However, the residuals indicate that the shape is not the right one.

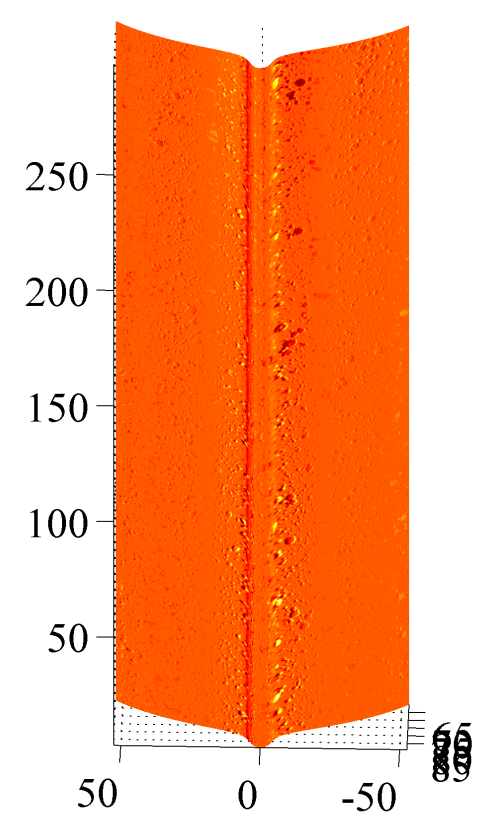

(a) $\operatorname{RMS}(r)=7.4 \%$

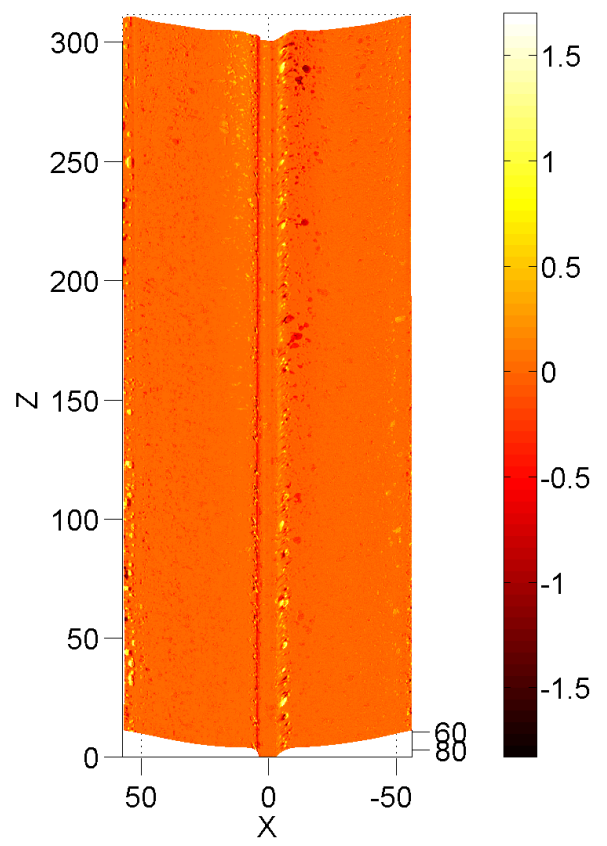

(b) $\operatorname{RMS}(r)=4.9 \%$

Figure 6: Initial shape used as first guess. The color map indicates the correlation residuals prior to (a), after (b) the calibration of the projection matrices

The second part of the calibration process is the measurement of the 3D shape of the surface of interest after which the RMS value of $r$ is brought down to $4.9 \%$ of the dynamic range of the reference pictures. It may be concluded that this result 
is satisfactory. However, a closer analysis of the reconstruction of the rib (Figure 7) shows spurious results. The circled zones show that loops are obtained. Although the obtained shape is closer to reality, this result is non physical.

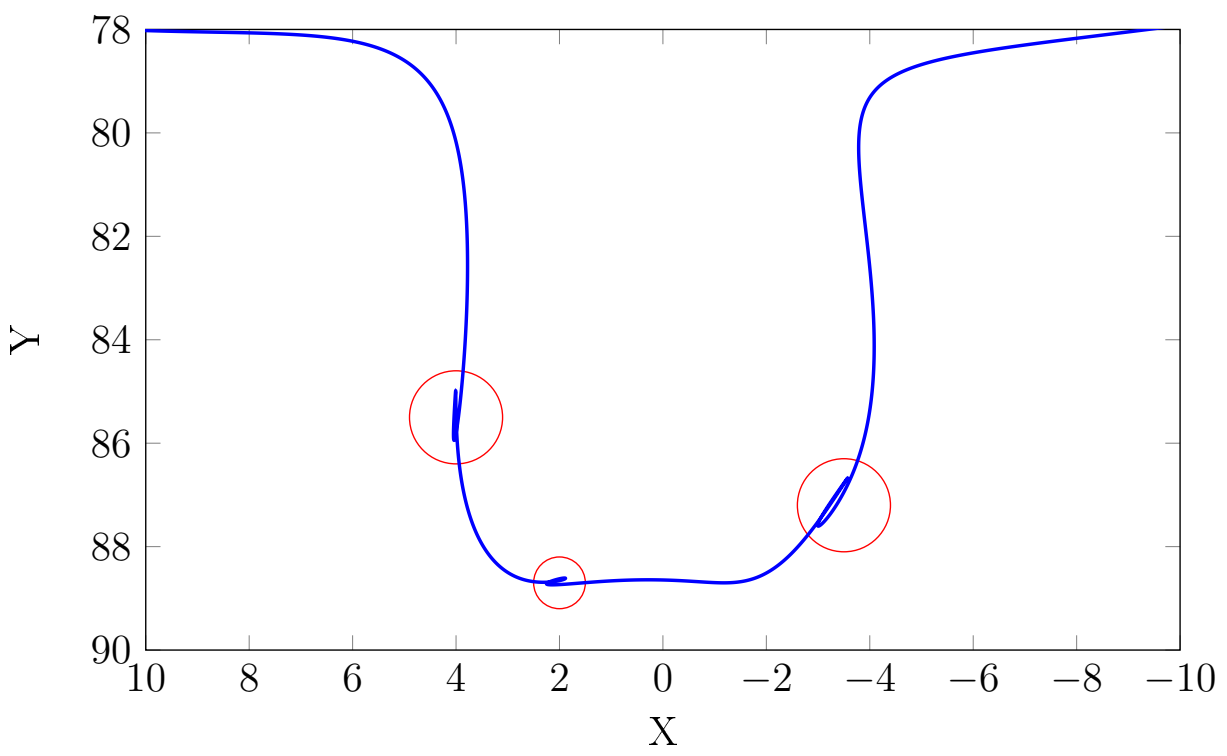

Figure 7: Close-up view of the reconstructed section. The areas of concern are circled in red

To understand the reason for the occurrence of such a phenomenon, the conditionning of the stereocorrelation matrix is analyzed. Because of the large number of poles needed to describe the rib (see Figure 5(b)) and visibility issues the conditioning of the stereocorrelation matrix $\left[\mathbf{C}_{\mathbf{P}}\right]$ indicates that the raw analysis is very sensitive to acquisition noise [19]. This is particularly true in the present case since part of the rib is not seen by both cameras. On the pictures of figures 4 (b) and (c) one notes that the left part of the rib is not visible by any of the two cameras. Since no information is available on this part of the rib, noise sensitivity is very important.

At this stage two decisions can be made. First, the registration is regularized as 
shown below. Second, the stereoscopic system may be repositioned in such a way that the reconstruction becomes well-posed. This route will be followed later on (Section 6).

\subsection{Regularized analysis}

Because of the poor conditioning of the stereocorrelation matrix $\left[\mathbf{C}_{\mathbf{P}}\right]$ observed above, a regularized approach is constructed using Tikhonov's procedure [20] to compute the corrections $\{\delta \mathbf{p}\}$ to the positions of the control points

$$
\left(\left[\mathbf{C}_{\mathbf{P}}\right]+\lambda[\mathbf{1}]\right)\{\delta \mathbf{p}\}=\left\{\mathbf{b}_{\mathbf{P}}\right\}-\lambda\left(\{\mathbf{p}\}-\left\{\mathbf{p}_{\mathbf{0}}\right\}\right)
$$

where $\{\mathbf{p}\}$ is the current estimate of the control point coordinates, $\left\{\mathbf{p}_{\mathbf{0}}\right\}$ the first guess of the control point positions (i.e., those of the nominal CAD model), and $\lambda$ is a parameter (to be chosen). Figure 8 shows that $\lambda$ acts as a cut-off length for a low-pass filter when the spectrum of eigen values of the stereocorrelation matrix is analyzed. The higher $\lambda$, the closer the reconstructed shape to its nominal model. Conversely, the lower $\lambda$, the more freedom is given to the stereocorrelation procedure to match the actual surface. However, it also becomes more sensitive to noise.

The proposed procedure therefore starts with values of $\lambda$ of the order of one hundredth of the maximum eigen value of $\left[\mathbf{C}_{\mathbf{P}}\right]$. After convergence of the minimization scheme, $\lambda$ is divided by 10 and the procedure is repeated until $\lambda$ reaches levels less than $10^{-10}$ times the maximum eigen value of $\left[\mathbf{C}_{\mathbf{P}}\right]$, which is very close to an unregularized procedure (Figure 8). This procedure allows the stereocorrelation code to be driven toward the minimum level without being trapped in secondary minima. This is all the more important when studying complex shapes. 


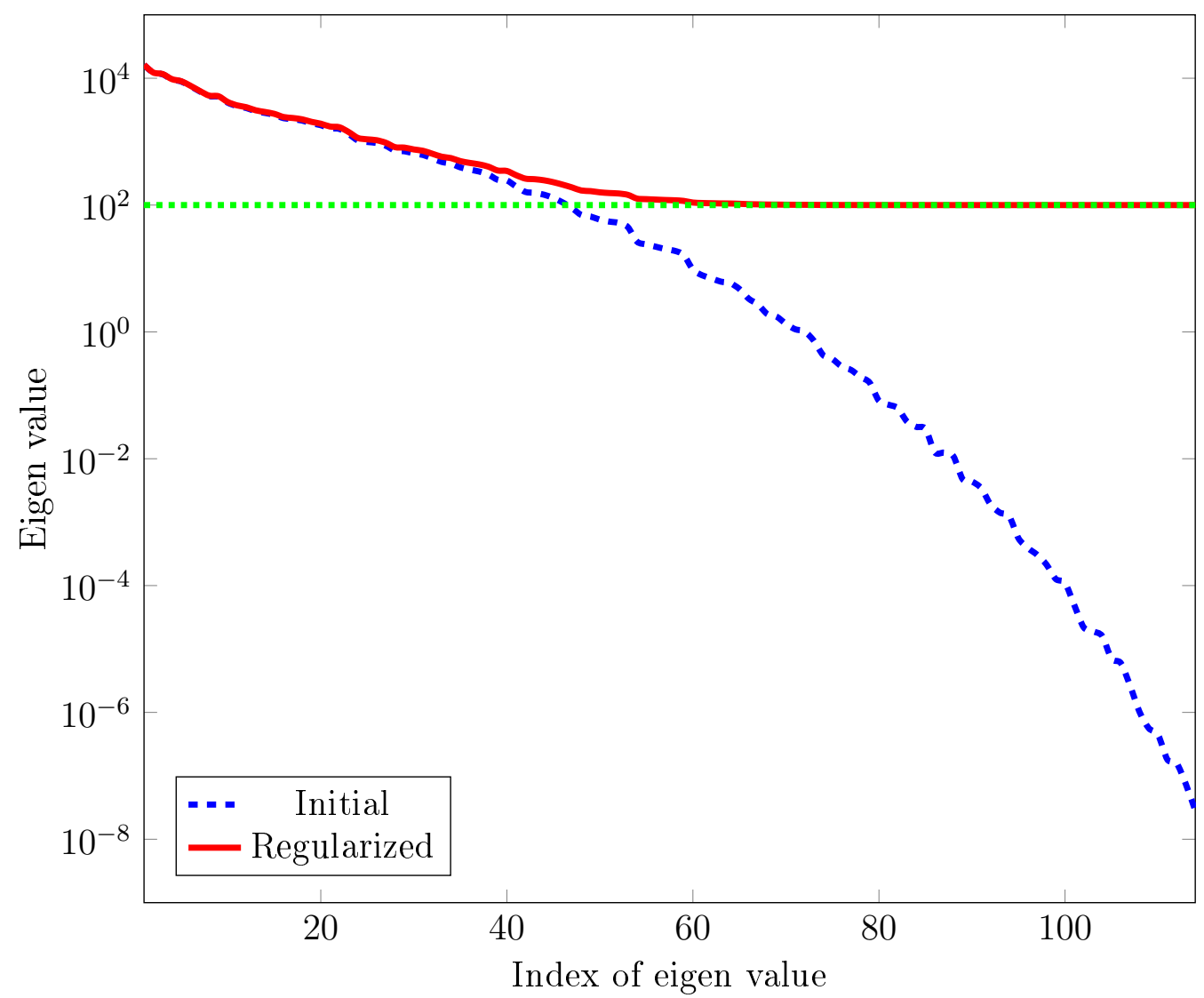

Figure 8: Spectrum of eigen values of the stereocorrelation matrix $\left[\mathbf{C}_{\mathbf{P}}\right]$ for the $3 \mathrm{D}$ reconstruction of the upper diaphragm. The horizontal dotted line corresponds to the current value of the regularization constant $\lambda$

Due to the Tikhonov regularization and relaxation, the algorithm currently requires more iterations (i.e., 78 in the present case) to reach convergence. Once the control point displacement amplitude is less than $10^{-5}$, the shape of the reconstructed surface is obtained directly in the NURBS formulation. An estimate of the real observed surface is obtained without being perturbed by uncertainties coming from the poor conditioning of high degree NURBS shapes (Figure 9). The RMS 
value of $r$ is equal to $2.9 \%$, to be compared with the level observed without a priori regularization (i.e., $4.9 \%$ ). This final result validates the proposed strategy.

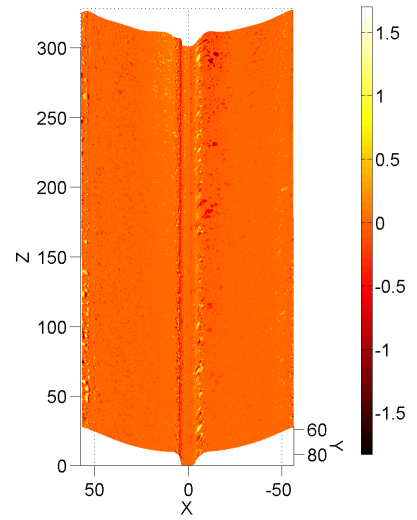

(a)

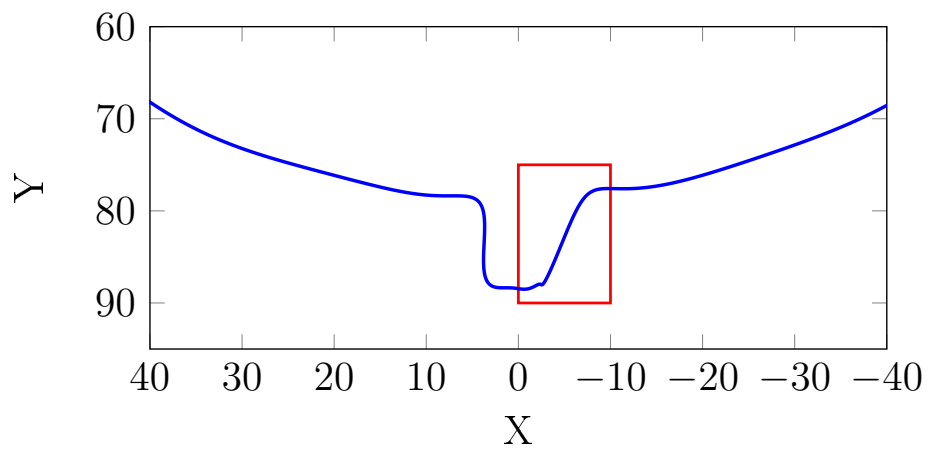

(b)

Figure 9: Measured 3D shape with regularization. (a) The color map indicates the correlation residuals after the 3D reconstruction. (b) Cross section of the reconstructed profile (the control points cannot be represented at the scale of the picture). The red box depicts the part with no visibility

The rib on the reconstructed surface has been sharpened (Figure 10). It fits better the real shape on one side. The other side cannot be reconstructed as accurately because it is not properly visible by one of the cameras (Figure 4). Thanks to the proposed approach, the problem is well-posed. Let us stress that for the hidden part the initial geometry is used as a substitute to the missing information (albeit it is not the exact shape). 


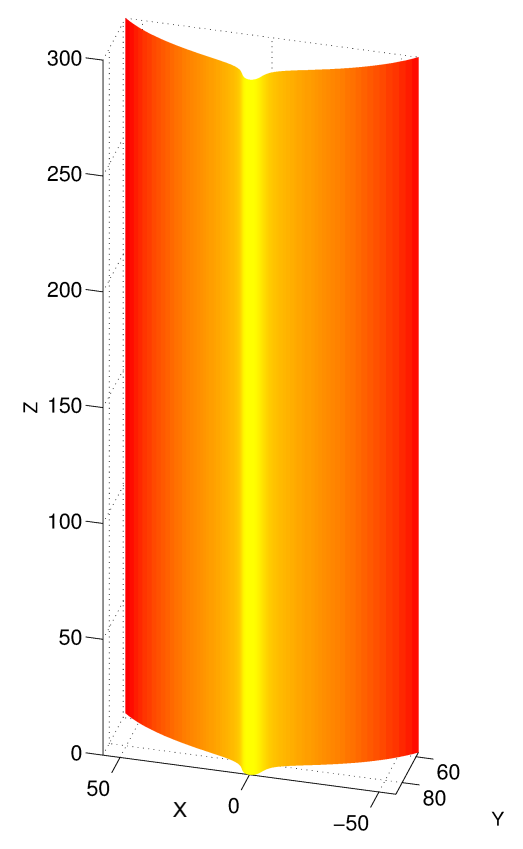

(a)

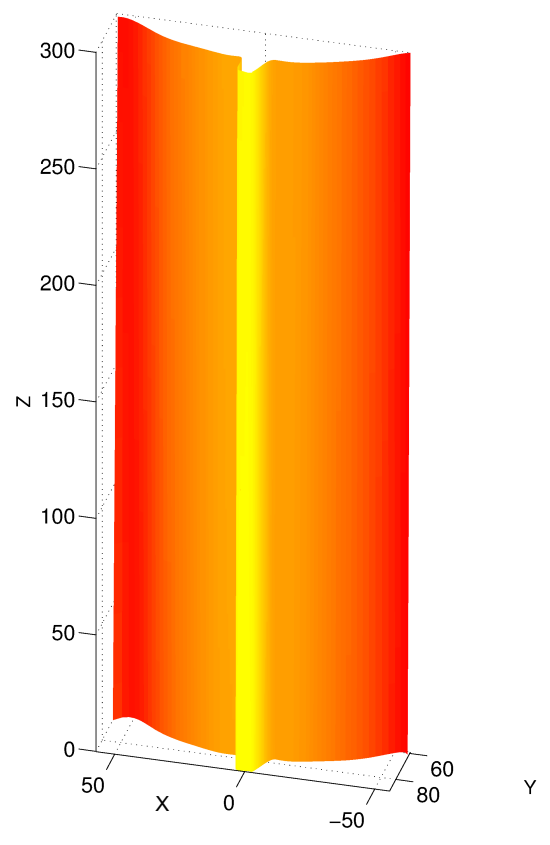

(b)

Figure 10: Initial (a) and reconstructed (b) shapes with regularization. The rib profile has been sharpened on the left side. The right side is not visible by one of the camera and thus cannot be reconstructed without the a priori knowledge. The color encoding indicates the height with respect to the underlying cylinder

\section{3D displacement measurements via isogeometric stereocorrelation}

In this section, the formulation of the 3D displacement field measurement via isogeometric stereocorrelation is introduced. For the sake of simplicity, it is assumed hereafter that the displacement fields are described in the same setting as the surface itself (i.e., the surface deformation is obtained by moving the control points). Let us stress that this point is not restrictive as an arbitrary dense set of control points 
could be considered if needed, notwithstanding the issue of actually computing the displacement. Other choices can be made (e.g., finite element descriptions [21, 22]). Within an isogeometric framework, motions of the control points will induce physical motions of any point of the analyzed surface that belongs to the parametric space. Because the surface texture can be transferred into the parametric space, all three components of the displacement are retrieved. A global approach to stereocorrelation consists of minimizing the functional $\eta$

$$
\begin{aligned}
\eta^{2}\left(\mathrm{~d}_{\mathbf{i j}}\right) & =\sum_{\text {ROI }}\left(g^{l}\left(\mathbf{x}_{n}^{l}\left(u, v, \mathrm{~d} \mathbf{P}_{\mathbf{i j}}\right)\right)-f^{l}\left(\mathbf{x}_{0}^{l}(u, v)\right)\right)^{2} \\
& +\sum_{\text {ROI }}\left(g^{r}\left(\mathbf{x}_{n}^{r}\left(u, v, \mathrm{~d} \mathbf{P}_{\mathbf{i j}}\right)\right)-f^{r}\left(\mathbf{x}_{0}^{r}(u, v)\right)\right)^{2}
\end{aligned}
$$

with respect to each coordinate motion $d \mathbf{P}_{\mathbf{i j}}$ of the control points $\mathbf{P}_{\mathbf{i j}}$ for the $n$ th picture pair. In the present case, $f^{l, r}$ denote the two pictures in the reference configuration, and $g^{l, r}$ the pictures in the deformed configuration (Figure 11). These control point motions induce (true) displacements in the left and right pictures (see Equation (13)). In the present case, $\mathbf{x}_{0}^{l, r}$ are the 2D positions of 3D points associated with the control points $\mathbf{P}_{\mathbf{i j}}(\mathbf{0})$ in the reference configuration, and $\mathbf{x}_{n}^{l, r}$ those in the deformed configuration (i.e., when the control points have moved by an amount $d \mathbf{P}_{\mathbf{i j}}$ such that $d \mathbf{P}_{\mathbf{i j}}=\mathbf{P}_{\mathbf{i j}}\left(\mathbf{u}_{\mathbf{n}}\right)-\mathbf{P}_{\mathbf{i j}}(0)$, see Figure 11). The minimization is again performed by resorting to a Newton-Raphson scheme. The following linear system is solved to determine the corrections $\{\delta \mathbf{p}\}$

$$
\left[\mathbf{C}_{\mathrm{d} \mathbf{P}}\right]\{\delta \mathbf{p}\}=\left\{\mathbf{b}_{\mathrm{d} \mathbf{P}}\right\}
$$

with

$$
\left[\mathbf{C}_{\mathrm{d} \mathbf{P}}\right]=\left[\mathbf{C}_{\mathrm{d} \mathbf{P}}^{\mathrm{l}}\right]+\left[\mathbf{C}_{\mathrm{d} \mathbf{P}}^{\mathbf{r}}\right] \text { and }\left\{\mathbf{b}_{\mathrm{d} \mathbf{P}}\right\}=\left\{\mathbf{b}_{\mathbf{P}}^{\mathbf{l}}\right\}+\left\{\mathbf{b}_{\mathbf{P}}^{\mathbf{r}}\right\}
$$


where $\left[\mathbf{C}_{\mathbf{P}}^{\mathbf{l}, \mathbf{r}}\right]$ denotes the $\left(n_{p} \times n_{p}\right)$ (left and right) stereocorrelation matrices

$$
\left[\mathbf{C}_{\mathbf{P}}^{\mathbf{l}, \mathbf{r}}\right]=\left[\mathbf{G}_{\mathbf{P}}^{\mathbf{l , r}}\right]^{\dagger}\left[\mathbf{G}_{\mathbf{P}}^{\mathbf{l}, \mathbf{r}}\right]
$$

$\left\{\mathbf{b}_{\mathbf{M}}^{\mathbf{l}, \mathbf{r}}\right\}$ the (left and right) stereocorrelation vectors

$$
\left\{\mathbf{b}_{\mathbf{P}}^{\mathbf{l}, \mathbf{r}}\right\}=\left[\mathbf{G}_{\mathbf{P}}^{\mathbf{l}, \mathbf{r}}\right]^{\dagger}\left\{\mathbf{r}^{\mathbf{l}, \mathbf{r}}\right\}
$$

$\left\{\mathbf{r}^{\mathbf{l}, \mathbf{r}}\right\}$ the (left and right) vectors gathering all correlation residuals

$$
r^{l, r}(u, v)=g^{l, r}\left(\mathbf{x}^{l}\left(u, v, \mathrm{~d} \mathbf{P}_{\mathbf{i j}}\right)\right)-f^{l, r}\left(\mathbf{x}_{0}^{r}(u, v)\right)
$$

and $\left[\mathbf{G}_{\mathbf{P}}^{\mathbf{l}, \mathbf{r}}\right]$ the $\left(n_{u} \times n_{v}\right) \times n_{p}$ matrix collecting all values of the scalar product of picture gradients $\boldsymbol{\nabla} f^{l, r}(u, v)$ by the sensitivity fields $\partial \mathbf{x}^{l, r} / \partial p_{i}(u, v)$.

This type of approach will be used hereafter to monitor the displacement field in a compression test of the upper diaphragm tube.

\section{Second Application}

In the present case, a new configuration of the stereoscopic system is considered. The two DSLR cameras are oriented in such a way that their plane of symmetry approximately coincides with that of the observed rib. The compression test is in displacement-controlled mode on a servohydraulic testing machine and a series of $2 \times 344$ pictures is shot during the first loading ramp. The whole stereocorrelation steps are followed to analyze the mechanical test. The final goal is to have access to the measured 3D displacement field of the region of interest. 


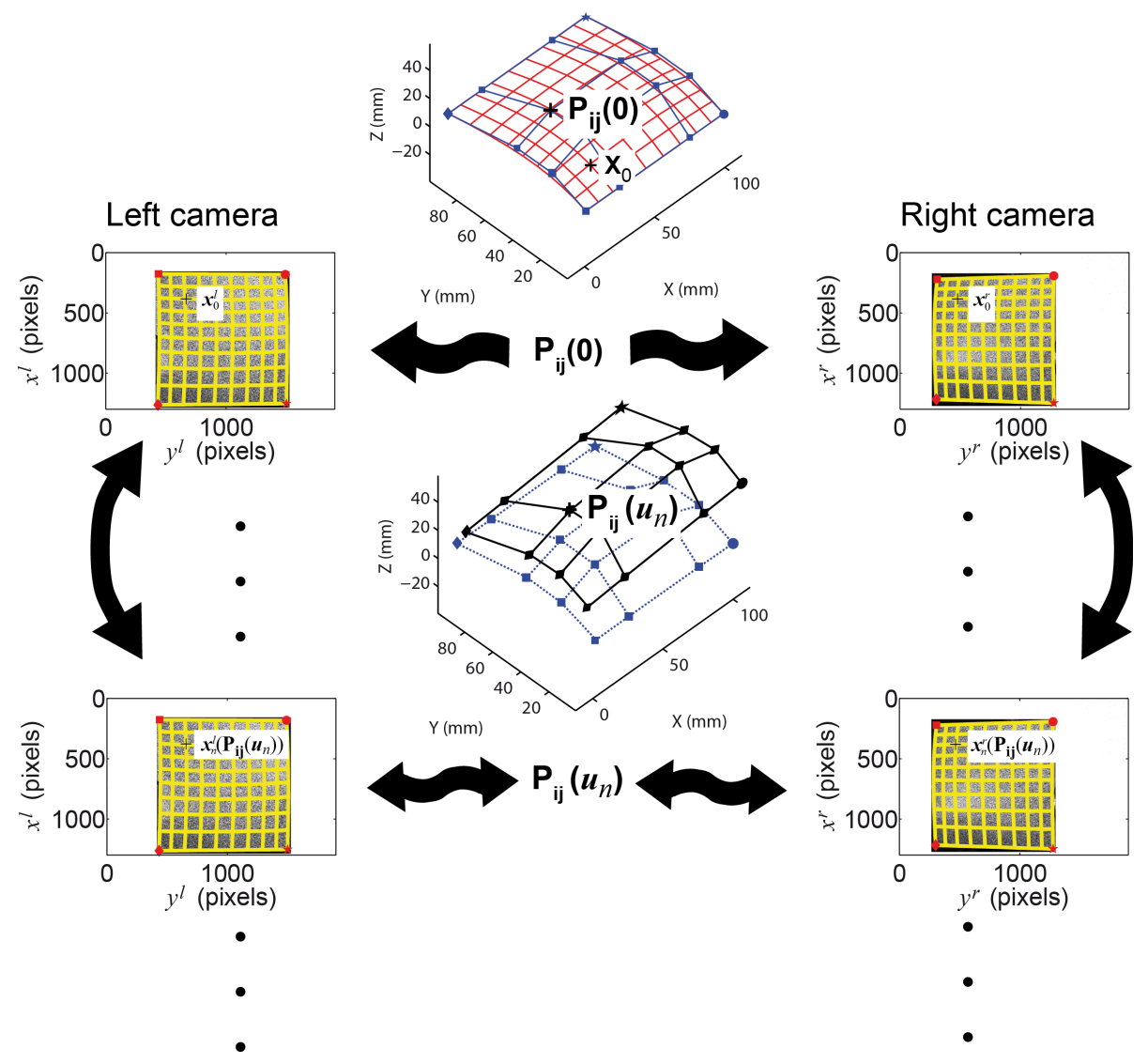

Figure 11: Measurement of 3D displacements via isogeometric stereocorrelation

\subsection{Self-Calibration}

The stereoscopic setup having been used for other purposes, the two-step calibration procedure is performed again. The initial guess of the 3D shape is still that shown in Figure 5(a) (i.e., the ribs are blunted as compared to what is expected). Figure 12(a) shows the initial correlation residuals prior to the first calibration step. Its RMS level is equal to $8.5 \%$. When the projection matrices are determined, it decreases down to $7.1 \%$. This level is of the same order as that observed in Section 4.1 . 


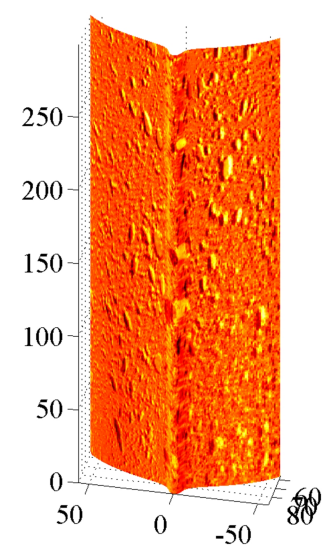

(a) $\operatorname{RMS}(r)=8.5 \%$

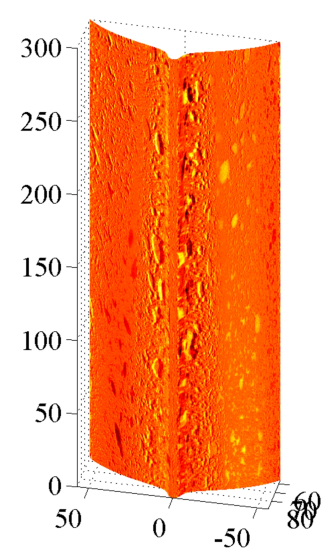

(b) $\operatorname{RMS}(r)=7.1 \%$

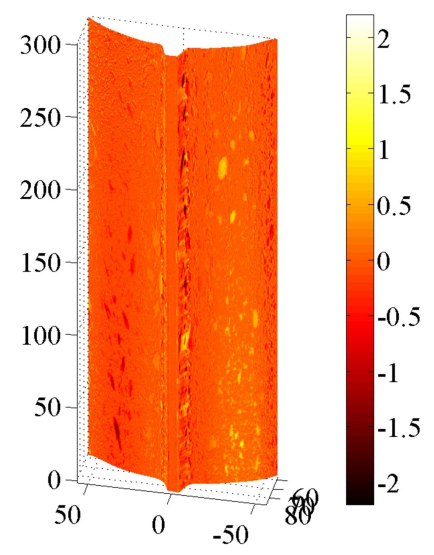

(c) $\operatorname{RMS}(r)=3.7 \%$

Figure 12: Correlation residuals prior to the calibration step (a), after the calibration of the projection matrices (b), and after the 3D shape measurement

From Figure 12(b), it is concluded that the remaining gap is mainly due to the fact that the actual ribs are sharper than what has been initially proposed to the stereocorrelation code. The second step of the calibration procedure thus consists of moving the control points to match as best as possible the actual surface. At the end of this second step, the correlation residuals have decreased to reach an RMS level of $3.7 \%$ (again close to what was achieved in Section 4.2). Figure 12 (c) shows that most areas have been successfully registered. There are still zones where the match is not perfect. One of the reasons is due to the fact that light reflection is not identical on the left and right cameras, and also that close to the ribs, information is still missing, to a lesser degree than before, on either of the cameras. Brightness and contrast corrections have not been considered in the present case. It is expected that the final residuals will be lowered even more with such an additional procedure [23, 24]. 
A Multiscale analysis is performed in this study to speed-up the convergence of the registration scheme. First, pictures are coarsened [25, 26]. The mean level of each $2 \times 2$-pixel block is used as a superpixel in the next scale. Second, the projection matrices are divided by the power of 2 corresponding to the scale change in order to be consistent with the new image size. Figure 13 shows the change of the RMS correlation residual as a function of the iteration number for scales no. 2 and 1 . The first iterations at scale 2 are regularized with $\lambda$ equal to one hundredth of the maximum eigen value of the stereocorrelation matrix. After convergence has been reached (i.e., after 5 iterations), the second part is solved with no regularization and an exponential convergence is observed. Similarly, for scale no. 1, when the system is regularized with a high weight, convergence is very fast (i.e., in one iteration). Had the multiscale approach not been used, many more iterations would have been used (i.e., 260 in the present case).

\subsection{D Displacement fields}

Figure 14(a) shows the history of the longitudinal displacement field. At the very beginning of the experiment, the displacements correspond to the cylinder set-up. After this initial phase, there is a uniform displacement gradient along the loading direction corresponding to uniaxial compression. Figure 14(b) shows the correlation residuals for the registration of the two image pairs corresponding to the maximum compression load. The RMS residual is equal to $1.1 \%$, which is very close to levels associated with acquisition noise. The random pattern is no longer visible thereby proving that the stereocorrelation algorithm has fully converged. The results are therefore deemed trustworthy. It is worth remembering that some areas close to 


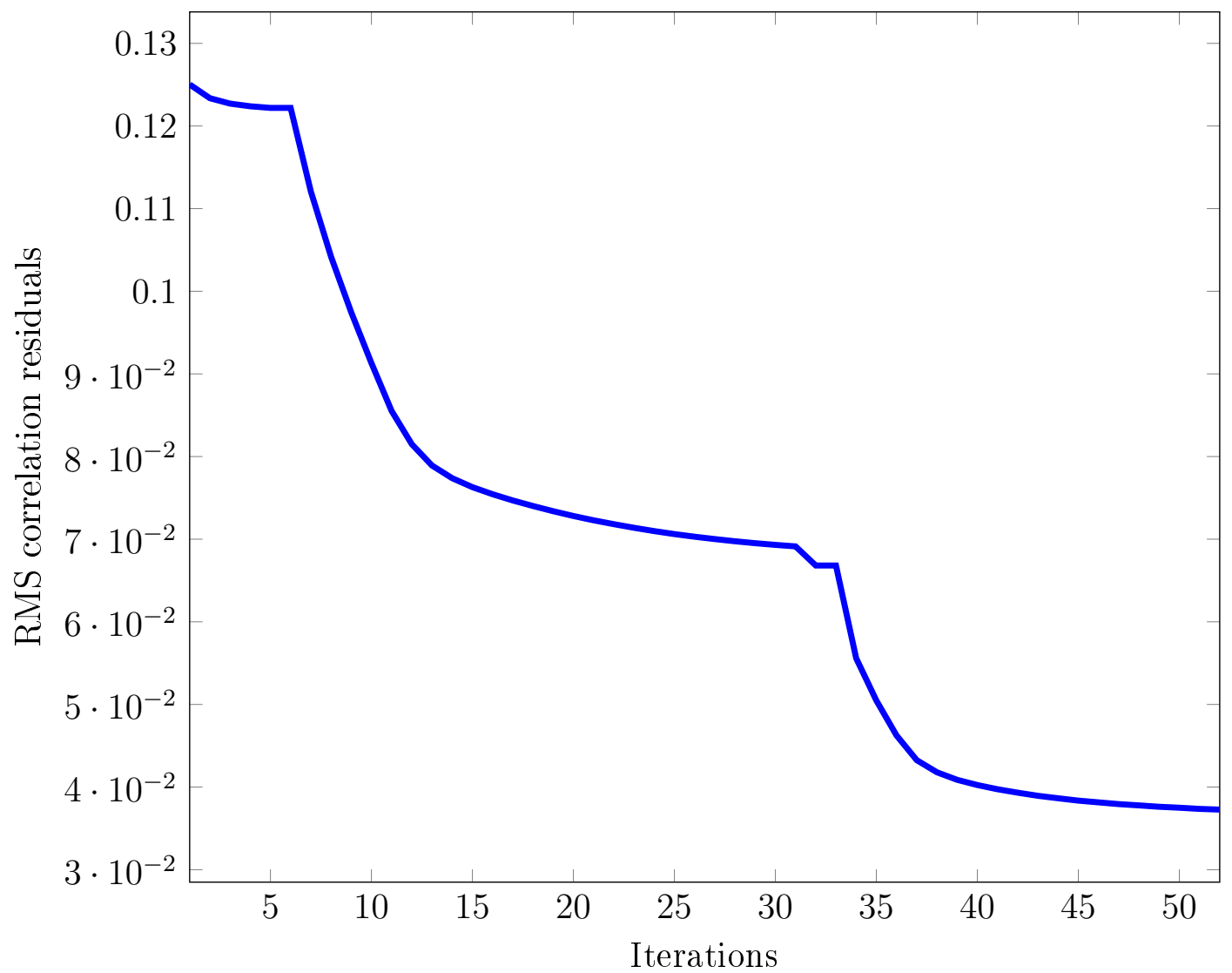

Figure 13: RMS correlation residual vs. iteration number for scale no. 2 (from iteration 1 to 23 ) and scale no. 1 (from iteration 23 to 52 )

the rib edges are not measurable per se. However, thanks to the global support of NURBS over the whole investigated surfaces, the corresponding displacements are extrapolated thanks to the continuity properties.

\section{Conclusion}

Isogeometric stereocorrelation has been used to analyze the deformation of an upper diaphragm of a landing gear. This global technique uses a mathematical model 

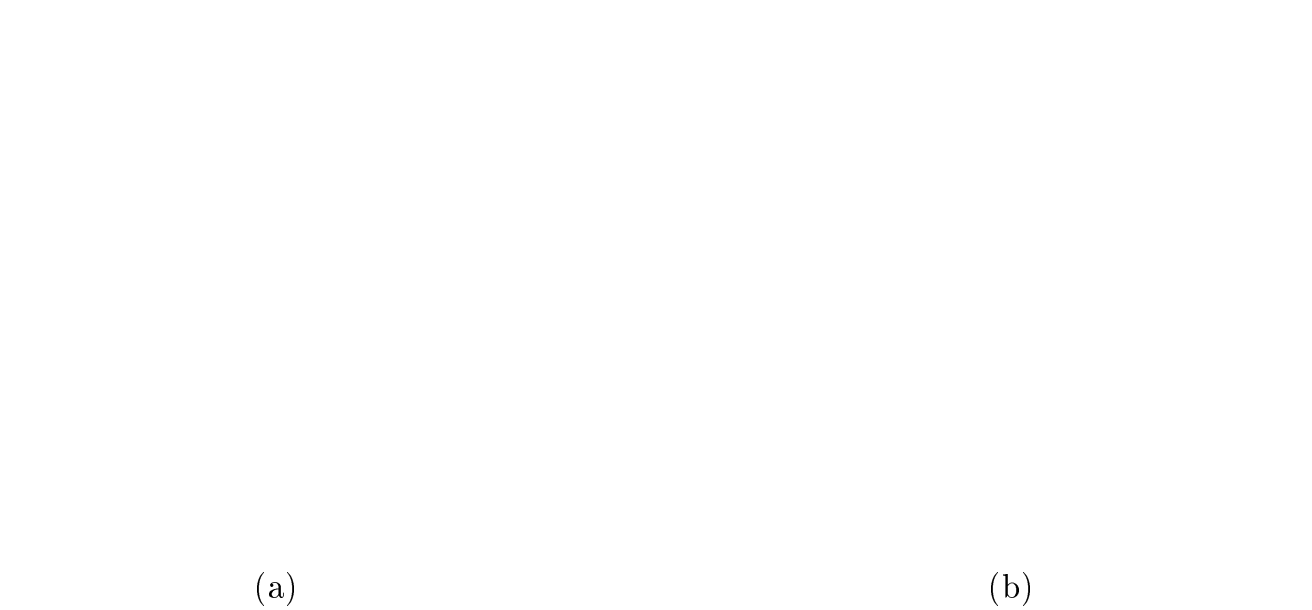

Figure 14: (a) Measured 3D displacements via regularized stereocorrelation, and (b) corresponding correlation residuals for the peak load. Online version: corresponding movie for 65 analyzed steps

of the surface of interest. In the present case NURBS patches are chosen. First, the calibration consists of two steps (i.e., calibration of the stereoscopic setup and measurement of the 3D shape). This type of calibration procedure is of special interest for structures for which classical procedures [9] may not be performed easily.

A regularization strategy has been implemented since parts of the surface of interest were not observed by both cameras of the stereo rig. Further, a multiscale approach allows to speed up the iterative scheme of global stereocorrelation. This is of particular interest when the initial guess is not very close to the reconstructed shape. As shown herein, these two features are very useful when dealing with complex 3D shapes.

Once the self-calibration is performed, the system is used to measure the 3D deformation of the analyzed surface during mechanical loading. The 3D displacement field is parameterized by the motion of the control points used to define the NURBS 
patches. The quality of the registrations is given by the correlation residuals, which are fields themselves so that local deviations can be monitored in addition to the global RMS level.

A complex shape with ribs on a cylinder has been reconstructed. This reconstruction does not need per se any subsequent interpolation stage, unless hidden parts exist on at least one camera. Otherwise, the NURBS representation provides a natural and convenient frame for regularization. This method can be applied to shapes of any complexity degree as far as a NURBS formulation is available and a regularization procedure used when the number of unknowns becomes too large to avoid spurious shape fluctuations, or when some small parts of the surface of interest is not observable by the camera(s).

Once validated thanks to the correlation residuals, the measured displacement fields give access to the computation of strain fields. The NURBS being very regular, they are very convenient to obtain the surface normals. Further, the determination of the displacement gradients can be evaluated analytically. It is worth noting that isogeometric measurements can be directly coupled with isogeometric simulations [27]. For instance, isogeometric model updating techniques can be considered for parameter identification purposes [23] or for validating the numerical procedures with realistic boundary conditions.

Last, the isogeometric setting can be extended to FE-based stereocorrelation, which uses meshes instead of NURBS patches. The principle is very close to the present approach. However, the regularity of the surface will not be as high as freeform descriptions. Consequently, more degrees of freedom will be needed to 
describe the surface of interest and regularized procedures may also be needed. These two global approaches belong to the class of dense multiview methods [28]. In the

case of FE-based approaches, the use of facets defined by triangular elements [29], Delaunay triangulation [30] or quadrilaterals [21, 22] are currently investigated.

\section{Acknowledgements}

The collaboration with Messier-Bugatti-Dowty and Snecma is gratefully acknowledged. This work was supported under the PRC Composites, French research project funded by DGAC, involving SAFRAN Group, ONERA and CNRS.

\section{References}

[1] S.S. Gorthi and P. Rastogi. Fringe projection techniques: Whither we are? Optics Lasers Eng., 48(2):133-140, 2010.

[2] P.F. Luo, Y.J. Chao, M.A. Sutton, and W.H. Peters. Accurate measurement of three-dimensional deformations in deformable and rigid bodies using computer vision. Exp. Mech., 33:123-132, 1993.

[3] F. Chen, G.M. Brown, and N. Song. Overview of three-dimensional shape measurement using optical methods. Opt. Eng., 39(1):10-22, 2000.

[4] M.A. Sutton, S.R. McNeill, J.D. Helm, and Y.J. Chao. Advances in TwoDimensional and Three-Dimensional Computer Vision, volume Topics in Appl. Phys., 77, pages 323-372. Springer, Berlin (Germany), 2000. 
[5] D. Garcia, J.J. Orteu, and L. Penazzi. A combined temporal tracking and stereocorrelation technique for accurate measurement of 3D displacements: Application to sheet metal forming. J. Mat. Proc. Tech., 125-126:736-742, 2002.

[6] B. Beaubier, J.E. Dufour, F. Hild, S. Roux, S. Lavernhe-Taillard, and K. Lavernhe-Taillard. CAD-based calibration of a 3D-DIC system: Principle and application on test and industrial parts. Exp. Mech., 54(3):329-341, 2014.

[7] A. Fusiello. Uncalibrated euclidean reconstruction: a review. Im. vis. comput., 18:555-563, 2000.

[8] J. Salvi, X. Armague, and J. Battle. A comparative review of camera calibrating methods with accuracy evaluation. Pattern Recog., 35:1617-1635, 2002.

[9] M.A. Sutton, J.J. Orteu, and H. Schreier. Image correlation for shape, motion and deformation measurements: Basic Concepts, Theory and Applications. Springer, New York, NY (USA), 2009.

[10] L. Piegl and W. Tiller. The NURBS Book - 2nd Edition. Springer, 1997.

[11] J.-E. Dufour, B. Beaubier, F. Hild, and S. Roux. CAD-based displacement measurements. Principle and first validations. Exp. Mech., 55(9):1657-1668, 2015.

[12] J. Harvent, F. Bugarin, J.J. Orteu, M. Devy, P. Barbeau, and G. Marin. Inspection of aeronautics parts for shape defect detection using a multi-camera system. In SEM conference, page 8p. SEM, 2008. 
[13] Dassault Systems. CATIA V5 R19, online documentation. http://www.3ds.com/products/catia/welcome/, 2008.

[14] Z. Zhang. A flexible new technique for camera calibration. IEEE Trans. Pattern Anal. Machine Intell., 22(11):1330-1334, 2000.

[15] O. Faugeras. Three-dimensional computer vision: a geometric viewpoint. MIT Press, Cambridge, MA (USA), 1993.

[16] S. Roux and F. Hild. Stress intensity factor measurements from digital image correlation: post-processing and integrated approaches. Int. J. Fract., 140(14):141-157, 2006.

[17] O.D. Faugeras, Q.T. Luong, and S.J. Maybank. Camera self-calibration: Theory and experiments. In Proc. 2nd ECCV, pages 321-334. Springer-Verlag, 1992.

[18] F. Hild and S. Roux. Digital Image Correlation, pages 183-228. Wiley-VCH, Weinheim (Germany), 2012.

[19] F. Hild and S. Roux. Comparison of local and global approaches to digital image correlation. Exp. Mech., 52(9):1503-1519, 2012.

[20] A.N. Tikhonov and V.Y. Arsenin. Solutions of ill-posed problems. J. Wiley, New York (USA), 1977.

[21] J. Réthoré, Muhibullah, T. Elguedj, M. Coret, P. Chaudet, and A. Combescure. Robust identification of elasto-plastic constitutive law parameters from digital images using 3D kinematics. Int. J. Solids Struct., 50(1):73-85, 2013. 
[22] J.E. Dufour, B. Beaubier, S. Roux, and F. Hild. Displacement measurement using CAD-based stereo-correlation with meshes. In ICEM conference, 2014.

[23] J.-E. Dufour, F. Hild, and S. Roux. Shape, Displacement and Mechanical Properties from Isogeometric Multiview Stereocorrelation. J. Strain Analysis, 50(7):470-487, 2015.

[24] A. Charbal, J.-E. Dufour, A. Guery, F. Hild, S. Roux, L. Vincent, and M. Poncelet. Integrated digital image correlation considering gray level and blur variations: Application to distortion measurements of ir camera. Opt. Lasers Eng., 78:75-85, 2016.

[25] F. Hild, B. Raka, M. Baudequin, S. Roux, and F. Cantelaube. Multi-scale displacement field measurements of compressed mineral wool samples by digital image correlation. Appl. Optics, IP 41(32):6815-6828, 2002.

[26] G. Besnard, F. Hild, and S. Roux. "Finite-element" displacement fields analysis from digital images: Application to Portevin-Le Chatelier bands. Exp. Mech., 46:789-803, 2006.

[27] J.A. Cottrell, T.J.R. Hughes, and Y. Bazilevs. Isogeometric Analysis: Toward Integration of CAD and FEA. Wiley, 2009.

[28] S.M. Seitz, B. Curless, J. Diebel, D. Scharstein, and R. Szeliski. A comparison and evaluation of multi-view stereo reconstruction algorithms. Proc. IEEE Conf. Computer Vision and Pattern Recognition, 1:519-526, 2006. 
[29] L. Dubreuil, J.-E. Dufour, Y. Quinsat, and F. Hild. FE-Based Shape Measurements with Stereocorelation. Submitted for publication, 2015.

[30] H.H. Vu, P. Labatut, J.P. Pons, and R. Keriven. High accuracy and visibilityconsistent dense multiview stereo. IEEE Trans. Pattern Anal. Machine Intell., 34(5):889-901, 2012. 